\title{
Randomized placebo-controlled clinical trial investigating the effect of antioxidants and a vasodilator on overall safety and residual hearing preservation in cochlear implant patients
}

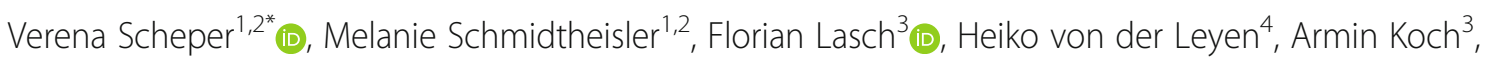
Jana Schwieger ${ }^{1}$, Andreas Büchner ${ }^{1,2}$, Anke Lesinski-Schiedat $^{1}$ and Thomas Lenarz ${ }^{1,2}$

\begin{abstract}
Background: The standard therapy for patients suffering from sensorineural hearing loss is cochlear implantation. The insertion of the electrode array into the cochlea, with potential mechanical trauma and the presence of this foreign body inside the cochlea, may lead to free radical formation and reduced blood perfusion of the cochlea which can result in a loss of residual hearing. Studies have suggested that a particular combination of the antioxidants vitamins $\mathrm{A}, \mathrm{C}$ and $\mathrm{E}$ as well as the vasodilator magnesium (together: ACEMg) may protect the residual hearing.

Methods: The potential protective effect of ACEMg on residual hearing preservation in cochlear implant (CI) patients was investigated in a single-centre, randomized, placebo-controlled, double-blind phase II clinical trial. Cl candidates with some residual hearing in low frequencies receiving MED-EL implants of different FLEX electrode array lengths were treated with ACEMg tablets or placebo respectively 2 days preoperatively and up to 3 months postoperatively. The study objective was to demonstrate that ACEMg is more efficacious than placebo in preserving residual hearing during cochlear implantation by comparing the hearing loss (change in hearing thresholds at 500 $\mathrm{Hz}$ from baseline) 3 months after the first fitting between the two treatment groups and to investigate the treatments' safety.

\footnotetext{
* Correspondence: scheper.verena@mh-hannover.de

'Department of Otolaryngology, Hannover Medical School, Carl-Neuberg-Str.

1, 30625 Hannover, Germany

${ }^{2}$ Cluster of Excellence Hearing4all, Oldenburg, Germany

Full list of author information is available at the end of the article
}

(c) The Author(s). 2020 Open Access This article is licensed under a Creative Commons Attribution 4.0 International License, which permits use, sharing, adaptation, distribution and reproduction in any medium or format, as long as you give appropriate credit to the original author(s) and the source, provide a link to the Creative Commons licence, and indicate if changes were made. The images or other third party material in this article are included in the article's Creative Commons licence, unless indicated otherwise in a credit line to the material. If material is not included in the article's Creative Commons licence and your intended use is not permitted by statutory regulation or exceeds the permitted use, you will need to obtain permission directly from the copyright holder. To view a copy of this licence, visit http://creativecommons.org/licenses/by/4.0/ The Creative Commons Public Domain Dedication waiver (http://creativecommons.org/publicdomain/zero/1.0/) applies to the data made available in this article, unless otherwise stated in a credit line to the data. 
(Continued from previous page)

Results: Fifty-one patients were included in the study, which had to be terminated before the recruitment goal was reached because of IMP-resupply mismanagement of one partner. In the intention-to-treat population, 25 patients were treated with ACEMg and 24 patients with placebo. The mean hearing loss at $500 \mathrm{~Hz}$ was ( \pm 15.84$)$ $30.21 \mathrm{~dB}$ (placebo) or ( \pm 17.56) $26.00 \mathrm{~dB}$ (ACEMg) 3 months after the initial fitting. Adjusting the postoperative hearing loss for the baseline residual hearing, planned electrode length and surgeon results in $8.01 \mathrm{~dB}$ reduced hearing loss in ACEMg-treated patients compared to placebo-treated ones. The safety analysis revealed that ACEMg was generally well-tolerated with adverse event frequencies below the placebo level.

Conclusion: This is the first clinical trial investigating a drug effect on residual hearing in $\mathrm{Cl}$ patients. These first-inman data may suggest that a perioperative oral administration of ACEMg is safe and may provide protection of residual hearing in $\mathrm{Cl}$ patients.

Trial registration: EU Clinical Trial Register No. 2012-005002-22. Registered on 6 December 2013. Funding: European Commission FP7-HEALTH-2012-INNOVATION-2.

Keywords: Free radical scavengers, Antioxidants, Cochlear implant, Hearing loss, Hearing pill, Hearing preservation, Residual hearing preservation, Vitamins

\section{Background}

The cochlear implant $(\mathrm{CI})$ is the standard treatment for uni- and bilateral severe and profound sensorineural hearing loss, both in adults and children. Around 600, 000 deaf individuals have already received cochlear implantations worldwide [1]. Audible signals are detected by a microphone, processed into an electrical signal and transmitted transcutaneous to an implanted receiver. The signal is decoded and delivered through an electrode array implanted into the scala tympani of the cochlea onto the auditory nerve (Fig. 1).
The whole frequency range of the acoustic signal is split into different frequency bands and allocated to the different electrode contacts, mimicking the physiological tonotopic organization of the cochlea [2]. As speech understanding with the CI has improved, indication criteria have been extended towards patients with loss of hearing in the high frequencies and residual low-frequency hearing. Thinner, shorter and more flexible atraumatic electrodes have been designed to match the recipient's individual cochlear anatomy to preserve its residual hearing [3].

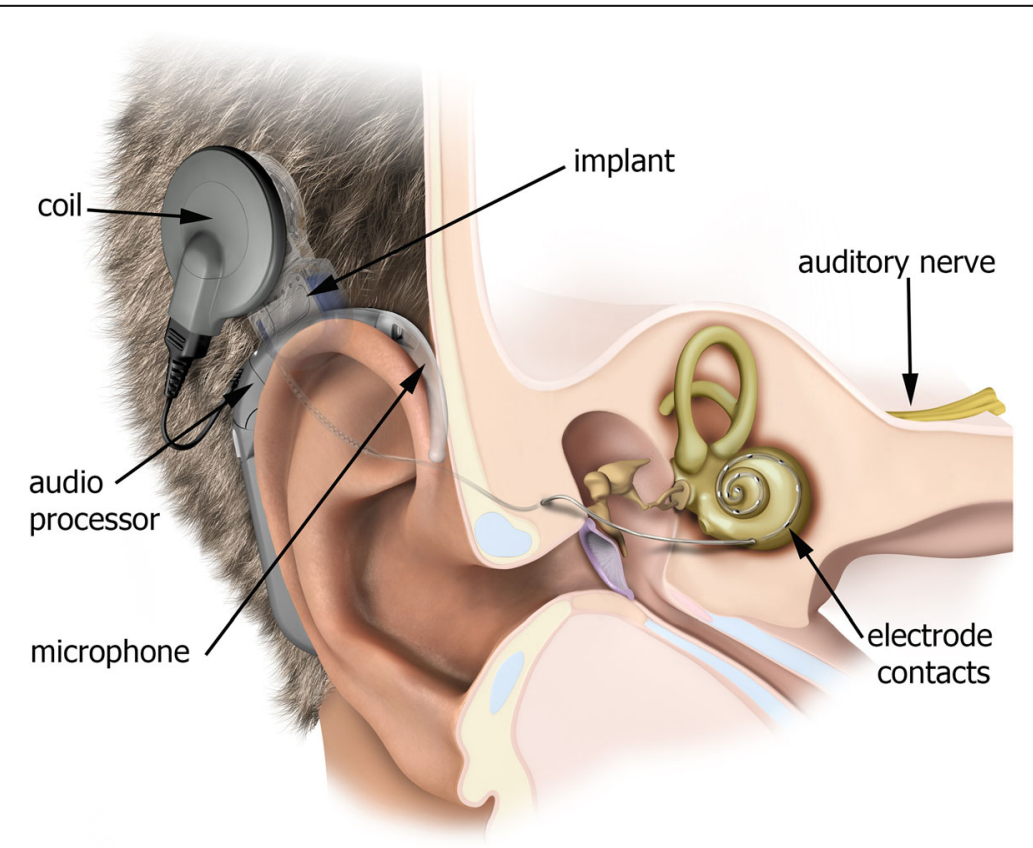

Fig. 1 Cochlear implant with microphone, processor, coil and lead wire. The electrode array is inserted into the inner ear (the illustration is reproduced with permission from MED-EL, Innsbruck, Austria) 
Even though the preservation of the residual hearing is possible, however, the preservation rates achieved are not satisfactory. Substantial acoustic hearing loss (mean threshold increase of $>30 \mathrm{~dB}$ ) is reported in $13 \%$ of cochlear implant patients receiving electric-acoustic stimulation (EAS) [4]. A moderate or partial loss of hearing (15 to $30 \mathrm{~dB}$ mean hearing loss) is described in $50.1 \%$ of the total patient population $(n=22)$ [5], and minimal loss of residual hearing (mean threshold shift $<15 \mathrm{~dB}$ ) has been reported, e.g. for $59 \%$ of patients 2 years after implantation [6] and for more than $43 \%$ of patients 1 year after implantation [7]. Suhling et al. report a median hearing loss of 15 to $32.5 \mathrm{~dB}$; shorter electrode arrays result in a better hearing preservation rate than using longer arrays [8].

The insertion of the electrode array into the cochlea has been shown to cause trauma to inner ear structures such as the basilar membrane, osseous spiral lamina and modiolus, and cascading molecular effects such as inflammation and oxidative stress $[9,10]$.

The loss of residual hearing after cochlear implantation is, as age-related hearing loss and noise-induced hearing loss, closely linked to the oxidative stress which is based on the formation of reactive oxygen species (ROS) [11]. The free radical formation is responsible for several types of damage to biological molecules present in the cochlea, as well as for the development of several other human diseases. In view of this, it would be desirable to be able to inactivate or reduce the formation of these free radicals, for example, by the use of antioxidants, agents that can inhibit or reduce damage in cells caused by free radicals. Foods, especially fruits, vegetables and legumes, also contain antioxidants, such as vitamins $\mathrm{C}, \mathrm{E}$ and $\mathrm{A}$; chlorophyllin; flavonoids; carotenoids; curcumin; and others that can restrict the dissemination of chain reactions and free radicalinduced damage. Evidence has accumulated indicating that dietary antioxidant supplementation has become a therapeutic strategy to prevent and/or to delay the risks of sensorineural hearing loss [12]. Experimental studies on the auditory system have demonstrated that antioxidants plus a vasodilator reduce both noise- and drug- (aminoglycoside)-induced inner ear pathology and hearing loss by $>75 \%[13,14]$. This formulation is $\beta$-carotene (converted in the body to vitamin $\mathrm{A}$ ), ascorbic acid (vitamin $\mathrm{C}$ ), trolox (vitamin $\mathrm{E}$ ) and the vasodilator magnesium. Together (ACEMg), they are remarkably effective in vivo to treat age-related hearing loss [15], noise-induced hearing loss $[13,16]$ and gentamycin-induced hearing loss [14]. Several reports indicate that free radicals play a key role in electrodeinduced cochlear damage $[9,17]$. The goal of ACEMg treatment is to reduce one important contributing factor, the stress of surgery and implantation of a foreign body that may negatively affect residual acoustic hearing in the implanted patient.
A meta-analysis aimed to evaluate randomized clinical trials to determine the effect of antioxidant supplementation on the auditory threshold in sensorineural hearing loss in patients of different age groups shows that there are no antioxidative therapies to preserve residual hearing after cochlear implantation [12].

The PROHEARING clinical trial (PROtect residual HEARING) is the first trial in humans to assess the efficacy of the antioxidative vitamins $\mathrm{A}, \mathrm{C}$ and $\mathrm{E}$ and the vasodilator magnesium to reduce hearing loss caused by cochlear implantation in CI patients with residual hearing.

\section{Methods}

This was a randomized, placebo-controlled, double-blind phase II clinical trial. The primary objective was to demonstrate that ACEMg is more efficacious than placebo in preserving residual hearing during cochlear implantation by comparing the hearing loss (air conduction hearing threshold postoperatively minus baseline threshold) at $500 \mathrm{~Hz} 3$ months after the first fitting. For this purpose, cochlear implantation candidates with low-frequency residual hearing were recruited. Treatments (ACEMg or placebo) were administered orally for 106 days. Patients were followed up 12 months post first fitting which is approximately 13 months postoperatively. The study was registered (EudraCT-No.: 2012-005002-22), and as demanded by the National Institute for Health and Clinical Excellence for clinical research publications to allow greater transparency of the study methods and measures used [18], the detailed study protocol was published [19]. The study was performed according to the Declaration of Helsinki (in its current version) and other applicable national legislation. The study was approved by the competent authority (BfArM), and the ethics committee of Hannover Medical School issued a favourable opinion.

\section{Subjects}

Study candidates were aged 18 years and older and suffered from pure/isolated cochlear-related hearing loss. This was proven with an objective audiological test battery before implantation. They had no or only little benefit from conventional hearing aids, defined as preoperative auditory speech understanding of less or equal to $60 \%$ in Freiburger monosyllables at $65 \mathrm{~dB}$ sound pressure level (SPL), best aided in the ear to be implanted. Their air conduction thresholds had to be better or equal than $85 \mathrm{~dB}$ hearing loss (HL) at $125 \mathrm{~Hz}, 90 \mathrm{~dB} \mathrm{HL}$ at $250 \mathrm{~Hz}$ and better or equal than $95 \mathrm{~dB} \mathrm{HL}$ at $500 \mathrm{~Hz}$ in the ear to be implanted. All patients received within routine CI surgery a Concerto or Synchrony implant from MED-EL (Innsbruck, Austria) (see the "Surgical technique and array choice" section). 


\section{Sample size}

The initial sample size calculation of this trial was based on historical data form the Hannover Medical School Department of Otolaryngology. In patients receiving different electrode lengths, a change in hearing thresholds at 500 $\mathrm{Hz}$ from baseline to 3 months after the first fitting (FF) of $15.6 \mathrm{~dB}$ with a standard deviation of $15.5 \mathrm{~dB}$ has been observed. Assuming equal standard deviations for ACEMgand placebo-treated patients and a difference in change of hearing threshold between the treatment groups, the sample size calculation was conducted using a two-group $t$ test with a two-sided type I error rate of 5\%. Under these assumptions, 150 patients per treatment group were calculated to be necessary to achieve a power of $80 \%$.

Due to recruitment difficulties caused by the inclusion and exclusion criteria, which may have been too stringent, the sample size target was reduced to 70 patients per group in a subsequent amendment resulting in a power of approximately $50 \%$ under the initial assumptions. However, for this phase II trial, the reduced sample size was judged acceptable to at least generate a signal whether ACEMg offers a promising treatment concept for the protection of residual hearing in CI patients [19].

\section{Blinding and randomization}

Patients were randomized 1:1 to ACEMg or placebo. The randomization was stratified by the length of the electrodes (short (=16 and $20 \mathrm{~mm}$ ) vs. medium $(24 \mathrm{~mm})$ vs. long $(28 \mathrm{~mm})$ as planned preoperatively (albeit the length of the electrode was finally determined during surgery). Randomization was performed centrally using a webbased randomization tool. For details, please see [19].

\section{ACEMg treatment}

All patients in the study received, per random allocation and double-blinded, treatment with ACEMg or placebo. The components of ACEMg are beta carotene (provitamine $\mathrm{A}$, converted into retinol; $18 \mathrm{mg}$ ), vitamin $\mathrm{C}$ (magnesium ascorbate; $500 \mathrm{mg}$ ), vitamin E (DL- $\alpha$-tocopherol acetate; $267 \mathrm{mg}$ ) and magnesium (magnesium citrate, magnesium ascorbate, magnesium stearate; $315 \mathrm{mg}$ ) (Table 1).

The patients received the daily dosage of ACEMg or placebo as $2 \times 3$ chewable tablets per day for a total of 106 days (first and last day 3 pills instead of 6) starting

Table 1 ACEMg doses per tablet and per daily dose in milligrams

\begin{tabular}{lll}
\hline Component & $\begin{array}{l}\text { Milligrams per tablet } \\
\text { (label claim) }\end{array}$ & $\begin{array}{l}\text { Milligrams per daily dose } \\
\text { (6 tablets per day) }\end{array}$ \\
\hline B-Carotene & 3.0 & 18 \\
Ascorbic acid & 83.33 & 499.98 \\
DL-a-tocopherol acetate & 44.5 & 267 \\
Magnesium & 52.5 & 315 \\
\hline
\end{tabular}

2 days before CI surgery. The study medication was also taken preoperatively at the day of surgery and after surgery for 103 further days.

\section{Compliance}

To quantify compliance with the study medication, serum vitamin E levels were measured in all randomized patients before, during (at surgery and 4 weeks postoperatively) and after the treatment period (month 3). Moreover, patients returned their treatment kits to the study site and provided information on their compliance via a short, self-administered questionnaire.

Patients under ACEMg treatment are only classified as compliant (and used for PP analysis), if their vitamin $\mathrm{E}$ level 4 weeks postoperatively was $\geq 20 \mu \mathrm{g} / \mathrm{ml}$.

Patients in the placebo group with postoperative vitamin E levels $\geq 20 \mu \mathrm{g} / \mathrm{ml}$ were judged as non-compliant to the study procedures, since they agreed with their informed consent not to use daily multi-vitamins or other supplements during the course of the study, but show vitamin $\mathrm{E}$ levels that are unrealistic without vitamin supplements.

Additionally, patients were classified as non-compliant if they state in a questionnaire a non-compliance $>50 \%$, and/or if the returned treatment kits contain $>50 \%$ of study medication, whereas missing bottles were considered as full bottles.

\section{Surgical technique and array choice}

Surgery was performed by nine experienced otosurgeons at Hannover Medical School. The technique was described previously [20]. Based on individual levels of residual hearing, the length of the electrode array was chosen by the surgeon. Patients were implanted with a Flex28 (28 mm array length), Flex24 (24 mm array length), Flex20 (20 mm array length) or Flex16 (a custom-made 16-mm array) (all from MED-EL, Innsbruck, Austria). The Flex20 and Flex16 were grouped as "Flex20" for the analysis. All electrodes were inserted using the round window approach.

\section{Pure-tone audiometry}

The primary objective was to compare the change in hearing thresholds at $500 \mathrm{~Hz}$ from baseline to 3 months after FF between the ACEMg- and placebo-treated groups.

To detect the frequency-specific acoustic hearing thresholds, air conduction thresholds were measured using a calibrated audiometer according to DIN EN 60318. The test method follows DIN ISO 8253 with headphones for air conduction and headset for bone conduction. Tested frequencies were 125, 250, 500 and $750 \mathrm{~Hz}$, and 1, 2, 4 and $8 \mathrm{kHz}$. Pure-tone audiometer limits were $95 \mathrm{~dB}$ at $125 \mathrm{~Hz}, 100 \mathrm{~dB}$ at $250 \mathrm{~Hz}$, and 110 $\mathrm{dB}$ at 500 to $1500 \mathrm{~Hz}$. All subjects underwent pure-tone 
air conduction audiometry preoperatively, at the first activation and 3, 6 and 12 months after the first fitting.

To determine the frequency-specific hearing loss, the difference between the preoperative threshold level and the respective threshold measured at each postoperative time point was calculated.

\section{First activation}

Four to 5 weeks after cochlear implantation, the audio processor OPUS 2 or DUET2, SONNET or SONNET EAS (MED-EL, Innsbruck, Austria) was first fitted in all patients.

\section{Data analysis}

\section{Analyses populations}

The intention-to-treat (ITT) population consists of all randomized patients who took the study medication at least once. Patients were analysed as randomized independently of their real treatment (ACEMg or placebo).

The per-protocol (PP) population comprises all patients complying with the protocol, i.e. patients who were eligible, received the treatment they were randomized to, were compliant to the study medication (see above) and procedures and had an outcome assessment at the respective time points.

In the safety population, all patients were analysed who received at least one dose of the study medication (verum or placebo). Patients were analysed as treated.

\section{Primary analysis}

The primary analysis was performed in the ITT population and was conducted as an ANCOVA model (= primary analysis model) including the treatment (ACEMg or placebo), the baseline residual hearing at $500 \mathrm{~Hz}$, the surgeon and the anticipated electrode length as covariates. The dependent variable was hearing loss at the implanted ear at $500 \mathrm{~Hz} 3$ months post first fitting (hearing loss $=3$ months post first fitting threshold minus 1-2 days preoperatively measured threshold) measured by air-conducted pure-tone audiometry. The frequency 500 $\mathrm{Hz}$ was chosen because it is the most important frequency for use of residual hearing, e.g. for voice recognition and speech in noise enhancement. If the patient reached at $500 \mathrm{~Hz}$, the upper air-conducted detection limit of the audiometer $(110 \mathrm{~dB})$ without hearing, the measurement was set to $120 \mathrm{~dB}$. Missing values were replaced by worst possible value per frequency (i.e. upper detection limit $+10 \mathrm{~dB})$.

\section{Results}

Based on the power calculation 140 patients $(n=70$ verum and $n=70$ Placebo) should have been enrolled [19]. The investigational medicinal products did run out of stability 18 months after project start. Since patients had to be supplied with study medication for 3 months, the actual total recruitment period was only 15 months. Due to financial insolvency of one of the consortium partners, resupply of study medication was not possible anymore and the trial had to be discontinued prematurely before the planned number of patients could be recruited. The authors believe that according to scientific standards it is necessary to publish data from discontinued clinical trials.

\section{Subjects}

Four hundred forty-seven patients were accessed for eligibility. A total of $N=51$ patients were enrolled until trial discontinuation, 26 in the ACEMg arm and 25 in the placebo arm. Figure 2 depicts in detail the reasons for not enrolling 396 patients. Two patients have been excluded from the efficacy analysis set (the ITT set): one from the placebo group because no cochlear implantation was conducted and one from the ACEMg group because he did not receive study medication. Therefore, $N=25$ patients were analysed in the ACEMg group and $N=24$ patients in the placebo group (both ITT set). Based on compliance measurements $N=11$ (ACEMg) and $N=12$ (placebo), patients were included in the perprotocol population (PP set) (Fig. 2).

The general medical history for all patients is stated in Additional file 1, supplement 1. Ear-specific medical history for the implanted ear is stated in Additional file 1, supplement 2. Supplement 3 shows the aetiology of the implanted ear. Concomitant medication for the ITT set summarized by events is stated in Additional file 1, supplement 4. Supplement 5 gives the hearing loss over time at $500 \mathrm{~Hz}$ with imputation of $110 \mathrm{~dB}$ if the upper detection limit is reached or $120 \mathrm{~dB}$ if the measurement is missing.

In Table 2, the baseline characteristics sex, age, implanted ear, electrode length and surgeon of the patients in the ITT set are stated by the treatment group. There are no clinically meaningful differences between the treatment groups. To investigate whether sex [21], age or baseline hearing at implantation did influence the treatment effect, a subgroup analysis was performed (see "Subgroup analysis" section).

\section{Hearing loss at $500 \mathrm{~Hz} 3$ months after the first fitting}

The primary efficacy parameter was hearing loss at the implanted ear at $500 \mathrm{~Hz} 3$ months post first fitting (hearing loss $=3$ months post first fitting threshold minus 1 2 days preoperatively measured threshold) measured by air-conducted pure-tone audiometry in the ear with a cochlear implant.

In the placebo group, the mean hearing loss (mean \pm SD) was increased by $30.21( \pm 15.84) \mathrm{dB}$ whereas in the ACEMg group, the mean hearing loss increase was 26.00 


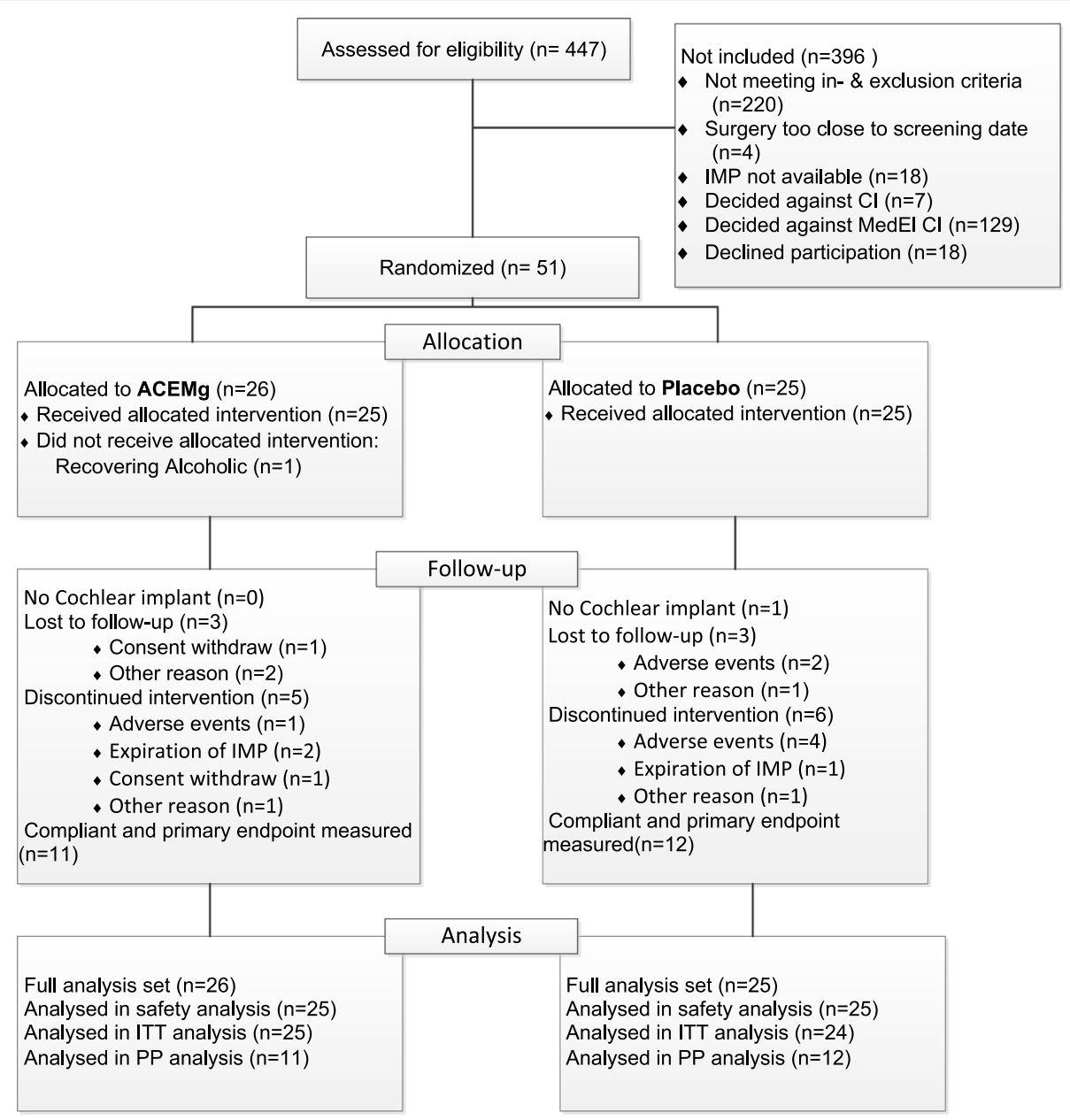

Fig. 2 Patient flow chart

$( \pm 17.56) \mathrm{dB}$. The superiority of ACEMg over placebo in preserving residual hearing during cochlear implantation could not be concluded because the $95 \%$ confidence interval for the difference in hearing loss between the ACEMg and placebo groups derived from the ANCOVA model used for primary analysis $(-12.95,4.65)$ includes zero $(p=0.3468$, Table 3$)$. Nevertheless, the estimate for ACEMg vs. placebo, adjusted for variables as listed in the ANCOVA model description, shows that descriptively, the hearing loss in the ACEMg group was on average $4.15 \mathrm{~dB}$ lower than in the placebo-treated patients (Table 3).

Figure 3 shows the individual hearing loss 3 months after the first fitting at $500 \mathrm{~Hz}$ by the treatment group, electrode length group and surgeon group.

Focusing on the used electrode lengths, it is obvious that the postoperative hearing loss (mean $\pm \mathrm{SD}$ ) of $11.25 \pm 12.50 \mathrm{~dB}$ in individuals implanted with a Flex 20 or Flex $16(n=4)$ is tremendously lower than in patients receiving a Flex $24(n=13 ; 32.69 \pm 17.87 \mathrm{~dB})$ or a Flex 28 $(n=32 ; 28.28 \pm 15.74 \mathrm{~dB})$. Statistical analyses revealed a significantly lower loss of residual hearing in Flex 20implanted patients compared to those receiving a Flex 24 or 28 (Table 3). The effect estimates for the different electrode lengths and the effect estimate for an overall effect of electrode length indicate that electrode length and hearing loss are not independent (ANCOVA derived $p$ value for an overall effect of electrode length: $p=$ 0.0029). The shorter the used electrode, the smaller is the hearing loss in this study. However, as secondary findings, these results are merely exploratory and do not allow causal inference because electrode length was not randomly assigned, but chosen preoperative by the surgeon. Out of the 49 patients receiving a CI, 29 were implanted by one surgeon (surgeon 2) and 20 patients were implanted by 8 other surgeons (Table 2 and Fig. 3). Since these 8 surgeons conducted at maximum 5 surgeries each, they were clustered for the statistical analysis. No statistically significant difference in hearing loss after surgery was detected between the patients based on surgeon $(p$ value $=0.66)$ even though patients being implanted by surgeon 2 exhibited a 2.03- $\mathrm{dB}$ lower mean 
Table 2 Baseline characteristics of the ITT set

\begin{tabular}{|c|c|c|c|}
\hline & Placebo, $N=24$ & ACEMg, $N=25$ & Total, $N=49$ \\
\hline \multicolumn{4}{|l|}{ Sex } \\
\hline Male & $9(37.5 \%)$ & $10(40.0 \%)$ & $19(38.8 \%)$ \\
\hline Female & $15(62.5 \%)$ & $15(60.0 \%)$ & $30(61.2 \%)$ \\
\hline$p$ value $\left(x^{2}\right)$ & & & 0.8575 \\
\hline \multicolumn{4}{|l|}{ Age } \\
\hline N & 24 & 25 & 49 \\
\hline Mean & 57.38 & 53.64 & 55.47 \\
\hline STD & 9.37 & 16.85 & 13.70 \\
\hline Mean difference & & & 3.74 \\
\hline$p$ value ( $t$ test) & & & 0.3413 \\
\hline \multicolumn{4}{|l|}{ Treated ear } \\
\hline Left & $7(29.2 \%)$ & $11(44.0 \%)$ & $18(36.7 \%)$ \\
\hline Right & $17(70.8 \%)$ & $14(56.0 \%)$ & $31(63.3 \%)$ \\
\hline$p$ value $\left(x^{2}\right)$ & & & 0.2816 \\
\hline \multicolumn{4}{|c|}{ Used electrode length } \\
\hline Flex 20 & $4(16.7 \%)$ & $3(12.0 \%)$ & $7(14.3 \%)$ \\
\hline Flex 24 & $4(16.7 \%)$ & $6(24.0 \%)$ & 10 (20.4\%) \\
\hline Flex 28 & $16(66.7 \%)$ & $16(64.0 \%)$ & $32(65.3 \%)$ \\
\hline$p$ value $\left(x^{2}\right)$ & & & 0.7700 \\
\hline \multicolumn{4}{|l|}{ Surgeon } \\
\hline Surgeon 1 & $2(8.3 \%)$ & $1(4.0 \%)$ & $3(6.1 \%)$ \\
\hline Surgeon 2 & $13(54.2 \%)$ & $16(64.0 \%)$ & $29(59.2 \%)$ \\
\hline Surgeon 3 & $2(8.3 \%)$ & $0(0.0 \%)$ & $2(4.1 \%)$ \\
\hline Surgeon 4 & $2(8.3 \%)$ & $3(12.0 \%)$ & $5(10.2 \%)$ \\
\hline Surgeon 7 & $1(4.2 \%)$ & $2(8.0 \%)$ & $3(6.1 \%)$ \\
\hline Surgeon 9 & $1(4.2 \%)$ & $0(0.0 \%)$ & $1(2.0 \%)$ \\
\hline Surgeon 10 & $1(4.2 \%)$ & $3(12.0 \%)$ & $4(8.2 \%)$ \\
\hline Surgeon 11 & $1(4.2 \%)$ & $0(0.0 \%)$ & $1(2.0 \%)$ \\
\hline Surgeon 12 & $1(4.2 \%)$ & $0(0.0 \%)$ & $1(2.0 \%)$ \\
\hline$p$ value $\left(x^{2}\right)$ & & & 0.5195 \\
\hline \multicolumn{4}{|c|}{ Surgeon (categorized) } \\
\hline Surgeon 2 & $13(54.2 \%)$ & $16(64.0 \%)$ & $29(59.2 \%)$ \\
\hline Other surgeons & $11(45.8 \%)$ & $9(36.0 \%)$ & $20(40.8 \%)$ \\
\hline$p$ value $\left(x^{2}\right)$ & & & 0.4839 \\
\hline
\end{tabular}

Table 3 Hearing loss (dB) 3 months post first fitting compared to baseline: results of the primary analysis ANCOVA model in the ITT set

\begin{tabular}{|c|c|c|c|c|}
\hline & Estimate & $\begin{array}{l}\text { Standard } \\
\text { error }\end{array}$ & $p$ value & $\begin{array}{l}95 \% \text { confidence } \\
\text { interval }\end{array}$ \\
\hline ACEMg - placebo & -4.15 & 4.3641140 & 0.3468 & $(-12.95,4.65)$ \\
\hline $\begin{array}{l}\text { Surgeon } 2 \text { - other } \\
\text { surgeons }\end{array}$ & -2.03 & 4.7141858 & 0.6688 & $(-11.54,7.48)$ \\
\hline Flex 24 - Flex 20 & 35.32 & 9.9561928 & 0.0010 & $(15.24,55.40)$ \\
\hline Flex 28 - Flex 20 & 42.14 & 12.2218146 & 0.0013 & $(17.49,66.79)$ \\
\hline Flex 28 - Flex 24 & 6.82 & 6.8177036 & 0.3231 & $(-6.93,20.56)$ \\
\hline $\begin{array}{l}\text { Baseline hearing at } \\
500 \mathrm{~Hz}(\mathrm{~dB})\end{array}$ & -0.48 & 0.1849977 & 0.0136 & $(-0.85,-0.10)$ \\
\hline
\end{tabular}

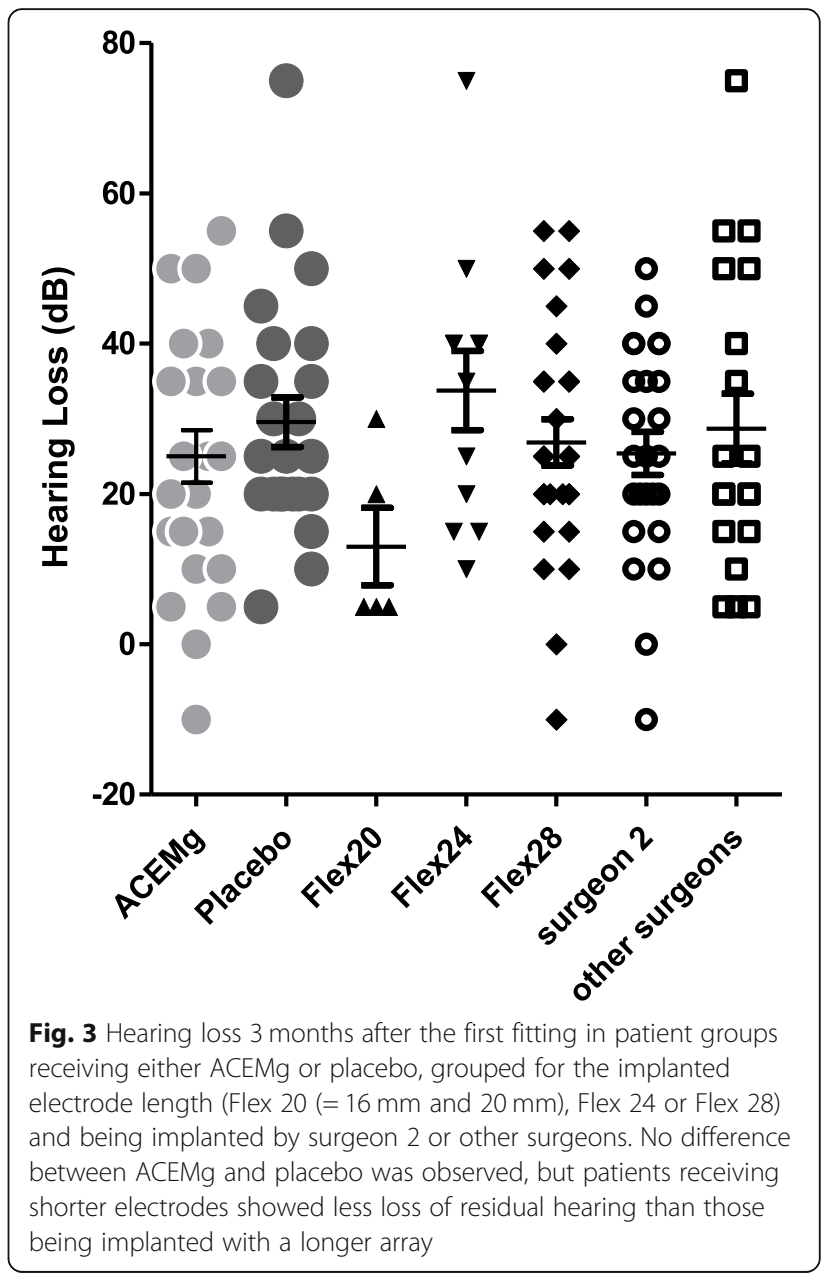

hearing loss than patients being implanted by the other surgeons (Table 3 ).

\section{Sensitivity analyses of primary analysis}

As a pre-defined sensitivity analysis, the primary analysis model (conducted as an ANCOVA model as described in the "Primary analysis" section) was applied in the PP population. Table 4 states the descriptive analysis of the primary endpoint in the PP population. In this population, the mean \pm SD hearing loss in the placebo group was $29.17( \pm 15.05) \mathrm{dB}$ and in the ACEMg group 23.64 $( \pm 18.99) \mathrm{dB}$. Here, the median hearing loss differed from $30 \mathrm{~dB}$ in the placebo-treated patients to $20 \mathrm{~dB}$ in the ACEMg group. Table 5 gives the results of the primary analysis model in the PP population. All sensitivity analyses are in line with the primary analysis result of the ITT population. The estimates for the treatment effect favour ACEMg over placebo without statistical significance and, thus, supporting the overall conclusion, that no superiority of ACMEg over placebo could be demonstrated in this prematurely determined study (Fig. 4). In the PP population, however, on average, the placebo- 
Table 4 Hearing loss (dB) 3 months post first fitting compared to baseline: a descriptive analysis of the primary endpoint-PP set

\begin{tabular}{llllllll}
\hline Subgroup & Number & Mean & $\begin{array}{l}\text { Std. } \\
\text { Dev. }\end{array}$ & Minimum & Median & Maximum \\
\hline Placebo & 12 & 29.17 & 15.05 & 5.00 & 30.00 & 55.00 \\
ACEMg & 11 & 23.64 & 18.99 & -10.00 & 20.00 & 55.00 \\
Flex 20 & 3 & 13.33 & 14.43 & 5.00 & 5.00 & 30.00 \\
Flex 24 & 6 & 28.33 & 15.71 & 10.00 & 27.50 & 50.00 \\
Flex 28 & 14 & 28.57 & 17.59 & -10.00 & 32.50 & 55.00 \\
Surgeon 2 & 16 & 25.94 & 14.97 & -10.00 & 30.00 & 50.00 \\
Other surgeons & 7 & 27.86 & 21.96 & 5.00 & 20.00 & 55.00 \\
\hline
\end{tabular}

treated patients have $8.01 \mathrm{~dB}$ more hearing loss than ACEMg-treated patients (Table 5), which is a clinically relevant difference.

\section{Subgroup analysis}

Subgroup analyses using the primary analysis model have been conducted by sex, age and baseline hearing measured by air-conducted audiometry at $500 \mathrm{~Hz}$ (Table 6). Regarding age, the study population was divided by the overall median age into two groups: age is (I) younger or equal to 55 years or (II) older than 55 years.

Regarding baseline hearing measured by air-conducted audiometry at $500 \mathrm{~Hz}$, the study population was divided by hearing threshold of $65 \mathrm{~dB}$ into two groups: hearing at $500 \mathrm{~Hz}$ is (i) less or equal to $65 \mathrm{~dB}$ or (ii) larger than $65 \mathrm{~dB}$.

Subgroup results regarding sex are in line with the primary analysis results (data not shown). The treatment effect estimates in both subgroups did not differ significantly from 0 . Thus, the primary analysis results interpretation did not change, and no inferential conclusions could be made.

The treatment effect estimates for ACEMg vs. placebo in the young and old subgroup were contrary. While in the subgroup of patients of less or equal to 55 years, ACEMg is estimated to be superior to

Table 5 Hearing loss (dB) 3 months post first fitting compared to baseline: results of the analysis of the primary endpoint-PP set

\begin{tabular}{lllll}
\hline & Estimate & $\begin{array}{l}\text { Standard } \\
\text { error }\end{array}$ & $p$ value & $\begin{array}{l}95 \% \text { confidence } \\
\text { interval }\end{array}$ \\
\hline ACEMg - placebo & -8.01 & 7.4323051 & 0.2961 & $(-23.69,7.67)$ \\
$\begin{array}{l}\text { Surgeon 2 - other } \\
\text { surgeon }\end{array}$ & -1.29 & 9.7399943 & 0.8963 & $(-21.84,19.26)$ \\
Flex 24 - Flex 20 & 26.40 & 15.9253500 & 0.1157 & $(-7.20,60.00)$ \\
Flex 28 - Flex 20 & 32.73 & 19.8918623 & 0.1182 & $(-9.23,74.70)$ \\
Flex 28 - Flex 24 & 6.33 & 10.5704737 & 0.5569 & $(-15.97,28.64)$ \\
Baseline & -0.28 & 0.3292886 & 0.4037 & $(-0.98,0.41)$ \\
\hline
\end{tabular}

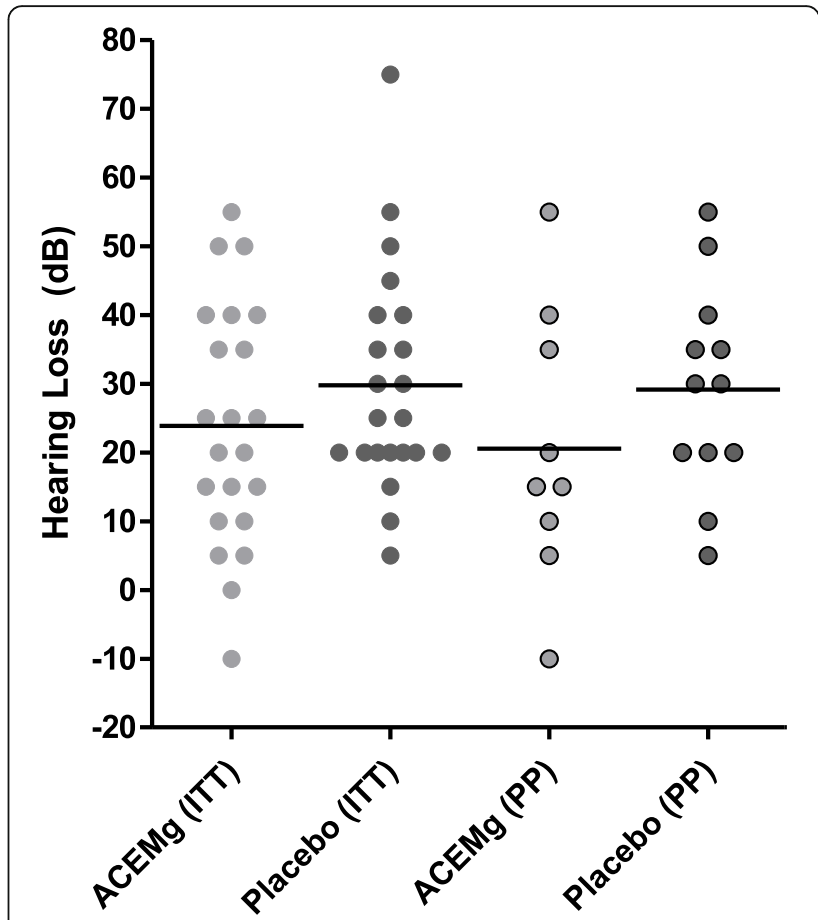

Fig. 4 Individual hearing loss 3 months after the first fitting in ACEMg- or placebo-treated patients of the ITT and PP population

placebo (8.24 dB lower hearing loss in ACEMg than in placebo), in the subgroup of patients older than 55 years, placebo is estimated to be superior over ACEMg (4.56 dB lower hearing loss in placebo than in ACEMg). However, both estimates did not differ significantly from 0 (Table 7).

Adjusting the primary analysis model for age (either as a continuous variable or a binary variable) did not change the analysis results regarding the treatment effect, and the estimated interaction term does not differ significantly from 0 (see Table 8 for continuous adjustment). Thus, the primary analysis results interpretation did not change, and no inferential conclusions could be made.

Subgroup analyses regarding baseline hearing at $500 \mathrm{~Hz}$ are given in Table 9 descriptively, and the results are given in Table 10. Subgroup analyses regarding baseline hearing show a smaller hearing loss in the ACEMg group in both subgroups. Additionally, a larger treatment effect can be observed in the subgroup with a baseline hearing threshold of less or equal to $65 \mathrm{~dB}$. However, in neither subgroup, the treatment difference is statistically significant. Subgroup results regarding baseline hearing are in line with the primary analysis. Nevertheless, it has to be mentioned that in this dataset, the effect of age (years) on hearing loss at $500 \mathrm{~Hz}$ is estimated to be 
Table 6 Hearing loss 3 months post first fitting compared to baseline: a descriptive subgroup analysis of the primary endpoint by age and residual hearing — ITT set

\begin{tabular}{|c|c|c|c|c|c|}
\hline Baseline factor & Subgroup & Treatment & Number & Mean HL & Std. Dev. \\
\hline \multirow[t]{4}{*}{ Age } & \multirow[t]{2}{*}{ Younger or equal to 55 years } & Placebo & 11 & 35.91 & 17.72 \\
\hline & & ACEMg & 15 & 21.33 & 17.97 \\
\hline & \multirow[t]{2}{*}{ Older than 55 years } & Placebo & 13 & 25.38 & 12.82 \\
\hline & & ACEMg & 10 & 33.00 & 15.13 \\
\hline \multirow[t]{4}{*}{ Residual hearing at $500 \mathrm{~Hz}$} & \multirow[t]{2}{*}{ Less or equal to $65 \mathrm{~dB}$} & Placebo & 9 & 37.22 & 21.23 \\
\hline & & ACEMg & 8 & 24.38 & 18.41 \\
\hline & \multirow[t]{2}{*}{ More than $65 \mathrm{~dB}$} & Placebo & 15 & 26.00 & 10.21 \\
\hline & & ACEMg & 17 & 26.76 & 17.67 \\
\hline
\end{tabular}

an increased hearing loss of $0.28 \mathrm{~dB}$ for each year of age at which the surgery is conducted (Table 8).

\section{Hearing loss over time}

The mean hearing loss increased over time in ACEMg- and placebo-treated patients from 25.00 respectively $29.57 \mathrm{~dB} 3$ months after the first fitting to 28.57 respectively $33.16 \mathrm{~dB} 12$ months after first fitting (Table 11, Fig. 5), resulting in a difference (ACEMg-placebo) of $-4.59 \mathrm{~dB}$. Over time, the number of patients conducting the respective study visits declined. In the ACEMg group, of the 25 patients being implanted (Fig. 2), 21 did finish their 12-month appointment. In the placebo group, from 24 implanted patients, only 19 conducted their 12 months visit (Table 11). When imputing $120 \mathrm{~dB}$ for missing measurements, as was conducted in the primary analysis only regarding hearing loss 3 months post first fitting, the difference (ACEMg - placebo) in hearing loss between ACEMg and placebo 12 months after the first fitting of $29.80 \mathrm{~dB}-36.25 \mathrm{~dB}$ is -6.45 $\mathrm{dB}$ (Additional file 1, supplement 5). As an additional sensitivity analysis, all time points were analysed simultaneously with a mixed model for repeated measures accounting for the correlation between the measurements of the same patient. In contrast to the analysis of the separate time points, here, the difference between ACEMg and placebo (ACEMg - placebo) 12 months post first fitting is estimated to be $-1.1 \quad(p=0.79)$, which indicates no perseverance of the observed difference 3 months post first fitting (see Additional file 1, supplement 6 for a short model description and respective results).
This result conflicts with the former results analysing the individual time points separately and applying different strategies for handling missing values (excluding missing values or imputing $120 \mathrm{~dB}$, respectively). Consequently, the high amount of missing values for the measurements 12 months post first fitting in the ITT set (19\%) and the above-described dependence of the estimated difference between ACEMg and placebo 12 months post first fitting on the imputation method used for missing values do not allow robust conclusions on the course and persistence of the treatment effect.

\section{Safety}

In total, 29 adverse events (AE) were registered, 14 of which were serious (supplement 7). Most adverse events were of grade 1 (68.9\%). $10.3 \%$ and $17.2 \%$ were of grades 2 and 3, respectively. One event (3.4\%) was of grade 4. No grade 5 adverse events were documented. Patients recovered completely from most AEs ( $n=26,89.7 \%)$. The outcome of two AEs was recovered with a sequel (hypertension and upper limb fracture) while the status of one $\mathrm{AE}$ remained unknown at database closure.

The number of both non-serious and serious adverse events (SAE) was numerically higher in the placebo group ( $n=18$ for AEs, $n=11$ for SAEs) than in the ACEMg group $(n=11$ and $n=3$ for AEs and SAEs, respectively).

Also, the number of AEs that resulted in the withdrawal of the study medication was numerically higher in the placebo group $(n=15)$ than in the ACEMg group $(n=8)$. Finally, the number of AEs

Table 7 Hearing loss 3 months post first fitting compared to baseline: results of the subgroup analysis by age-IT set

\begin{tabular}{llllll}
\hline Subgroup & Effect & Estimate & Standard error & $p$ value & $95 \%$ confidence interval \\
\hline Younger or equal to 55 years & ACEMg - placebo & -8.24 & 7.7076232 & 0.2980 & $(-24.31,7.84)$ \\
Older than 55 years & ACEMg - placebo & 4.56 & 6.5936026 & 0.4981 & $(-9.35,18.48)$ \\
\hline
\end{tabular}


Table 8 Hearing loss 3 months post first fitting compared to baseline: effect estimate for ACEMg vs. placebo, surgeon, electrode length and age from ANCOVA model adjusted by age as a continuous covariate

\begin{tabular}{lllll}
\hline Effect & Estimate & Standard error & $p$ value & $95 \%$ confidence interval \\
\hline ACEMg - placebo & -3.14 & 4.3410215 & 0.4735 & $(-11.90,5.62)$ \\
Surgeon 2 - other surgeons & -2.65 & 4.6540781 & 0.5715 & $(-12.05,6.74)$ \\
Flex 24 - Flex 20 & 33.99 & 9.8300736 & 0.0013 & $(14.15,53.83)$ \\
Flex 28 - Flex 20 & 35.54 & 12.7415605 & 0.0079 & $(9.82,61.25)$ \\
Flex 28 - Flex 24 & 1.55 & 7.5051096 & 0.8378 & $(-13.60,16.69)$ \\
Baseline & -0.37 & 0.1935072 & 0.0605 & $(-0.76,0.02)$ \\
Age & 0.28 & 0.1782778 & 0.1254 & $(-0.08 ; 0.64)$ \\
\hline
\end{tabular}

that were assessed as possibly causally related to the study medication $(n=4$ : ear pain, tympanic membrane perforation, vertigo and cerebrospinal fluid leakage) by the reporter was also higher in the placebo group $(n=10)$ than in the verum group $(n=4)$.

\section{Discussion}

\section{Efficacy}

Most recently, Wanna et al. detected hearing preservation rates of $38 \%$ 2-3 weeks postoperatively decreasing to $18 \%$ at about 19 months post-surgery [22]. Since many variables may affect the residual hearing directly during and after cochlear implantation as well as months and years after surgery, the reason for hearing loss may be multifactorial. Electrode design, surgical approach, and inflammation have all been implicated as potentially important variables.

In this study, we focused on one possible aspect leading to post-implantation hearing loss. We concentrated on the free radical formation and the possible reduction using antioxidative vitamins and a vasodilator to increase cochlear blood flow. In this placebocontrolled single-centre clinical trial, the effect of orally administered vitamins $\mathrm{A}, \mathrm{C}$ and $\mathrm{E}$ together with magnesium on residual hearing in $\mathrm{CI}$ patients and the dependency of hearing loss after cochlear implantation on age at implantation, residual hearing status, sex or surgeon was evaluated.

As described above, the study did not reach the recruitment goal due to IMP-resupply mismanagement of one partner, resulting in 51 included patients $(=36 \%)$ of the planned sample size of 140 .

Nevertheless, the analysis of the primary objective in the recruited ITT population showed that the mean hearing loss measured at $500 \mathrm{~Hz} 3$ months post first fitting compared to baseline in the placebo group was $30.21( \pm 15.84) \mathrm{dB}$ whereas in the ACEMg group, the hearing loss was $26.00( \pm 17.56) \mathrm{dB}$. There was no statistically significant difference, but a $4.15-\mathrm{dB}$ smaller mean hearing loss was observed in the ACEMg-treated patients compared to the placebo group. Clinically, in general, a difference of $4.15 \mathrm{~dB}$ in hearing ability is a relevant improvement. This tendency of residual hearing preservation 3 months after the first fitting was still detectable 1 year after the implantation with $36.25 \mathrm{~dB}$ mean hearing loss in patients receiving placebo and $29.80 \mathrm{~dB}$ mean hearing loss in ACEMg-treated patients, even though the main amount of radicals is released immediately after implantation. This suggests that the initial intake of ACEMg within the first 3 months after the first fitting may have a prolonged protective effect on residual hearing. As in the placebo group, the hearing loss was ongoing in the ACEMg group within the first 12 months after the first fitting. For the ACEMg group, some analysis suggested a lower extent of hearing loss over time than the loss over time detected in the placebo group. However, it needs to be noted that for $18 \%$ of the patients in the ITT set, the measurement of residual hearing 12 months after the first fitting was missing, and the prolonged protective effect could

Table 9 Hearing loss 3 months post first fitting compared to baseline: descriptive subgroup analysis by baseline hearing at $500 \mathrm{~Hz}$ air-conducted audiometry (>65 dB or $\leq 65 \mathrm{~dB}$ ) - IT set

\begin{tabular}{|c|c|c|c|c|c|c|c|}
\hline \multicolumn{8}{|c|}{ Hearing loss 3 months post-fitting compared to baseline-ITT set } \\
\hline Subgroup & Treatment & N & Mean & Std. Dev. & Minimum & Median & Maximum \\
\hline \multirow[t]{2}{*}{ Less or equal to $65 \mathrm{~dB}$} & Placebo & 9 & 37.22 & 21.23 & 5.00 & 40.00 & 75.00 \\
\hline & ACEMg & 8 & 24.38 & 18.41 & 5.00 & 17.50 & 55.00 \\
\hline \multirow[t]{2}{*}{ More than $65 \mathrm{~dB}$} & Placebo & 15 & 26.00 & 10.21 & 10.00 & 25.00 & 45.00 \\
\hline & ACEMg & 17 & 26.76 & 17.67 & -10.00 & 25.00 & 50.00 \\
\hline
\end{tabular}


Table 10 Hearing loss 3 months post first fitting compared to baseline: results of ANCOVA model applied for subgroup analyses by baseline hearing at $500 \mathrm{~Hz}$ air-conducted audiometry (> $65 \mathrm{~dB}$ or $\leq 65 \mathrm{~dB}$ ) — IT set

\begin{tabular}{llllll}
\hline Subgroup & Effect & Estimate & Standard error & $p$ value & 95\% confidence interval \\
\hline Less or equal to 65 dB & ACEMg - placebo & -11.46 & 9.1677502 & 0.2374 & $(-31.63,8.72)$ \\
& Surgeon 2 - other surgeons & -3.12 & 9.6611576 & 0.7529 & $(-24.38,18.15)$ \\
& Flex 24 - Flex 20 & 23.26 & 13.8967484 & 0.1224 & $(-7.33,53.85)$ \\
& Flex 28 - Flex 20 & 27.69 & 17.8852439 & 0.1498 & $(-11.68,67.06)$ \\
More than 65 dB & Flex 28 - Flex 24 & 4.43 & 12.9790722 & 0.7393 & $(-24.14,33.00)$ \\
& ACEMg - placebo & -1.89 & 4.4766332 & 0.6767 & $(-11.07,7.30)$ \\
& Surgeon 2 - other surgeons & -0.23 & 4.8327054 & 0.9623 & $(-10.15,9.69)$ \\
& Flex 28 - Flex 24 & 13.09 & 8.2152402 & 0.1227 & $(-3.77,29.95)$ \\
\hline
\end{tabular}

not be replicated in sensitivity analyses applying different missing data assumptions. Consequently, further studies have to be conducted to proof this potential benefit of ACEMg.

Since the likelihood of residual hearing preservation in cochlear implantation is related to medical history and surgical factors such as duration of hearing loss, aetiology, route of implantation (round window approach vs. cochleostomy), and device-related factors such as shape, length and flexibility of the array, it has to be concluded that there are multiple factors which affect the residual hearing. Zanetti et al. correlated age, side of implant, implant model and type of cochleostomy to hearing loss after cochlear implantation and reported that the mean threshold variations did not reach statistical significance for any of these variables. A slight trend in favour of better residual hearing preservation in children vs. adults was seen, especially at lower frequencies [23]. Conform to Zanetti's results, we did not find a dependency of hearing loss and ear to be implanted (data not shown). But there was a strong dependency of hearing preservation and electrode length used with smaller hearing loss for shorter electrodes and higher loss for longer ones. This is in accordance with other studies reporting good hearing preservation when using shorter electrodes [8].

In the placebo group, the hearing loss at $500 \mathrm{~Hz}$ determined 3 months $(30.21 \pm 15.84 \mathrm{~dB})$ or 12 months $(36.25 \pm 19.63 \mathrm{~dB})$ after the first fitting was in the range of hearing preservation rates reported previously $[4,8]$. We detected a not significant tendency of progressive hearing loss over time in placebotreated patients, which was already reported by others [6, 24-26].

In our study, the subjects enrolled did not substantially differ regarding their demographical data between the treatment groups. The mean age in the ACEMg group was 3.74 years lower than in the placebo group ( $p=$ $0.3413)$. Clinically, 3.7 years are judged as irrelevant, but since the residual hearing may correlate with age due to age-related hearing loss, the hearing performance of the patients may decline with age. Therefore age is a relevant factor and it has to be avoided to have a mismatch in age between the treatment groups. The potential influence of this imbalance was investigated by subgroup analyses with age added as either continuous or dichotomized (by median age) factor to the primary analysis

Table 11 Hearing loss at $500 \mathrm{~Hz}$ at all time points compared to baseline

\begin{tabular}{|c|c|c|c|c|c|c|c|}
\hline Time point & Treatment & Number & Mean & Std. Dev. & Minimum & Median & Maximum \\
\hline \multirow[t]{2}{*}{3 months post-fitting } & Placebo & 23 & 29.57 & 15.88 & 5.00 & 25.00 & 75.00 \\
\hline & ACEMg & 24 & 25.00 & 17.19 & -10.00 & 25.00 & 55.00 \\
\hline \multirow[t]{2}{*}{6 months post-fitting } & Placebo & 24 & 31.04 & 17.69 & 5.00 & 27.50 & 75.00 \\
\hline & ACEMg & 24 & 25.83 & 16.79 & -5.00 & 25.00 & 55.00 \\
\hline \multirow[t]{2}{*}{9 months post-fitting } & Placebo & 21 & 33.81 & 16.50 & 15.00 & 30.00 & 75.00 \\
\hline & ACEMg & 22 & 26.82 & 17.08 & -5.00 & 25.00 & 55.00 \\
\hline \multirow[t]{2}{*}{12 months post-fitting } & Placebo & 19 & 33.16 & 17.34 & 5.00 & 30.00 & 75.00 \\
\hline & ACEMg & 21 & 28.57 & 16.89 & 5.00 & 25.00 & 55.00 \\
\hline
\end{tabular}

The calculation is based on the available measurements at each time point applying the following imputation: If the upper detection limit is reached (110 dB), $120 \mathrm{~dB}$ is imputed. Missing values not due to measurement limits are not imputed 


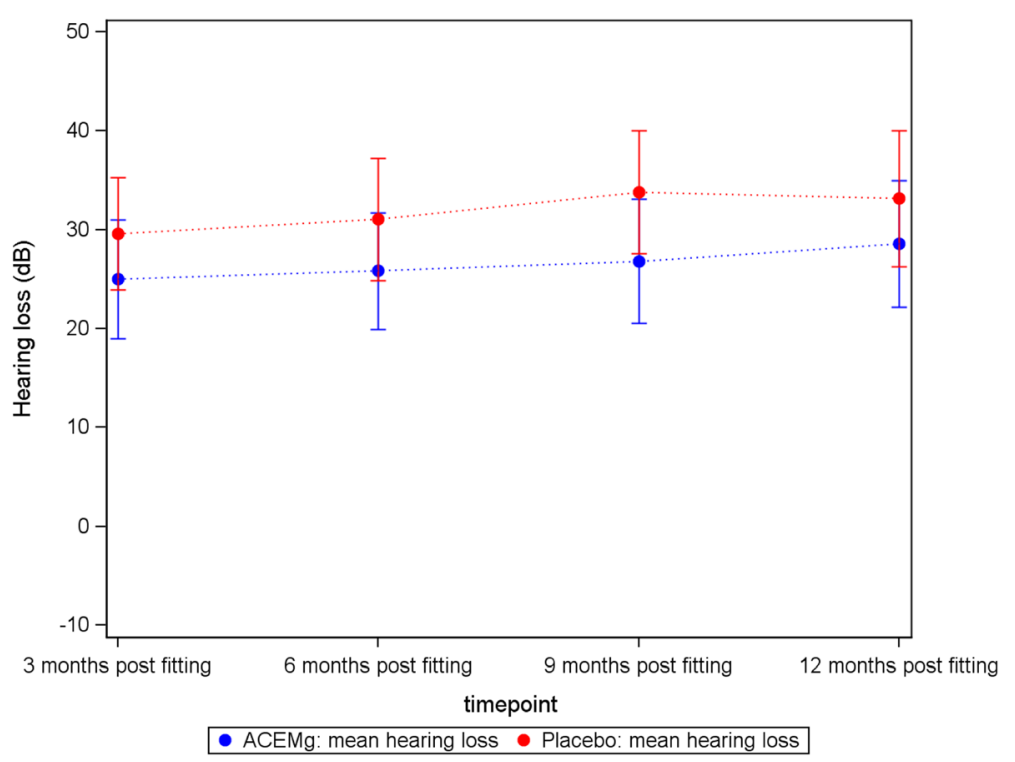

Fig. 5 Mean hearing loss over time in the ACEMg- and placebo-treated groups. The calculation is based on the available measurements at each time point applying the following imputation: If the upper detection limit is reached (110 dB), $120 \mathrm{~dB}$ are imputed. Missing values not due to measurement limits are not imputed. At all observed time points, the hearing loss in the placebo group was higher compared to the hearing loss detected in the ACEMg group. In both groups, the hearing loss increased over time

model. Without showing statistically significant differences, the subgroup analyses estimate ACEMg to be superior to placebo in patients aged 55 years or younger with an effect size of $8.24 \mathrm{~dB}$ lesser hearing loss in ACEMg-treated patients. This effect is turned to the opposite in patient older than 55 years, where placebo shows a not significantly reduced hearing loss with an estimated effect of $4.56 \mathrm{~dB}$. It is well known that, in general, the free radical formation is higher in older people [27]. We hypothesize that the additional oxidative stress due to the electrode insertion [9] may lead to a summating of free radicals resulting in an oxidative stress overload in older patients which cannot be treated by the ACEMg doses regiment used in our study.

\section{Safety}

The number and spectrum of AEs fall within the expected range for the population studied. Also, the number of AEs that were likely to be related to the study procedure is to be expected in the context of the surgical procedure. No increased risk compared to placebo could be observed in ACEMg-treated patients. ACEMg was generally well-tolerated with $\mathrm{AE}$ frequencies at (or below) the placebo level. No actions for safety had to be taken during the PROHEARING trial.

\section{Conclusion}

The data of this prematurely discontinued trial show a tendency that ACEMg therapy in patients aged 55 years and younger starting directly before cochlear implantation and lasting for about 100 days may lead to a better hearing preservation 3 months after the first fitting and lasting for at least 13 months after surgery. We hypothesize that a food supplement of the metabolic imbalances during and after surgery by antioxidative and vasodilatative supplementation may improve the benefit some patients will have by receiving a CI. Further studies need to be conducted addressing this topic to verify the promising preliminary data of this trial.

\section{Supplementary information}

Supplementary information accompanies this paper at https://doi.org/10. 1186/s13063-020-04522-9.

Additional file 1. Includes the general medical history for all patients in
the ITT-set as Supplement 1. Ear specific medical history for the im-
planted ear is stated in Supplement 2 of the additional file 1. Supplement
3 shows the aetiology of the implanted ear. Concomitant medication for
the ITT-set summarized by events is stated in Supplement 4 . Supplement
5 gives the hearing loss over time at $500 \mathrm{~Hz}$ with imputation of $110 \mathrm{~dB}$ if
the upper detection limit is reached or $120 \mathrm{~dB}$ if the measurement is
missing. Supplement 6 reports the hearing preservation measured by air
conducted audiometry at $500 \mathrm{~Hz}$ including all timepoints analyzed in the
modified ITT population using a mixed model with change from baseline
as dependent variable and treatment, baseline hearing threshold, sur-
geon, electrode length, electrode length - visit interaction and visit-
treatment interaction as fixed effects. The mixed model for repeated
measures was conducted adjusting for either the planned electrode
length (Supplement Table 6.1) as was done in the primary analysis model,
or by adjusting for the used electrode length (Supplement Table 6.2). The
estimated mean hearing loss at all time points with 95\%-confidence in-
tervals based on the mixed model for repeated measures adjusted for
the planned electrode length is illustrated in supplementary figure 6. AEs
are listed in supplement 7.




\section{Abbreviations}

ACEMg: Vitamins A, C and E and magnesium; AE: Adverse event; Cl: Cochlear implant; EAS: Electric-acoustic stimulation; FF: First fitting; HL: Hearing loss; ITT: Intention-to-treat; MAX: Maximum; PP: Per-protocol; ROS: Reactive oxygen species; SAE: Serious adverse event; SD: Standard deviation; SPL: Sound pressure level

\section{Acknowledgements}

The authors thank Cornelia Batsoulis (MED-EL) for her scientific support and colleagues at the Deutsches Hörzentrum Hannover (DHZ) for their support in data acquisition. We thank all patients for participating in this study. We appreciate their help and willingness to perform more detailed postoperative measurements which is sometimes uncomfortable or annoying. Their contribution to this important research is invaluable for helping to develop strategies to improve the cochlear implant outcome.

\section{Authors' contributions}

VS, MS, ALS and TL were responsible for the clinical part of the protocol. AK and FL performed the statistical planning of the study, based on clinical expertise from VS, TL, ALS and AB. HVDL was responsible for the trial management. The clinical part of the study was conducted by MS, VS, JS, $A L S$ and $A B$ with TL being the principal investigator. FL and AK performed the biostatistics analysis. VS, FL and HVDL were responsible for the overall assembly of the manuscript. All authors read, edited and approved the final manuscript.

\section{Funding}

This work was supported by the European Commission FP7-HEALTH-2012INNOVATION-2, project: "A novel micronutrient-based strategy to prevent hearing impairments: test and road to market for age-related hearing loss and preservation of residual hearing (PROHEARING)".

\section{Availability of data and materials}

The datasets used and analysed during the current study are available from the corresponding author on reasonable request.

\section{Ethics approval and consent to participate}

This study was reviewed by The German Bundesinstitut für Arzneimittel und Medizinprodukte (BfArM), application number 4039192, and registered on December 6, 2013, with protocol amendment version 3.0 from August 19, 2014. EudraCT number: 2012-005002-22 (https://www.clinicaltrialsregister.eu/ ctr-search/search?query=2012-005002-22). All patients gave written consent to participate in this study.

\section{Consent for publication}

Not applicable

\section{Competing interests}

The authors declare that they have no competing interests.

\section{Author details}

'Department of Otolaryngology, Hannover Medical School, Carl-Neuberg-Str. 1, 30625 Hannover, Germany. ${ }^{2}$ Cluster of Excellence Hearing4all, Oldenburg, Germany. ${ }^{3}$ Institute for Biostatistics, Hannover Medical School, Carl-Neuberg-Str. 1, 30625 Hannover, Germany. ${ }^{4}$ Hannover Clinical Trial Center, Carl-Neuberg-Str. 1, 30625 Hannover, Germany.

Received: 28 February 2020 Accepted: 16 June 2020

Published online: 14 July 2020

\section{References}

1. Foundation TE. Cochlear implant information sheet. 2019; Available from: www.earfoundation.org.uk.

2. Lenarz T, Scheper V. Preserving Residual Hearing in Cochlear Implant Patients. in "Free Radicals in ENT Pathology". Eds.: Miller J, LePrell CG, Rybak L. Springer; 2015. ISBN 978-3-319-13473-4.

3. Dhanasingh A, Jolly C. An overview of cochlear implant electrode array designs. Hear Res. 2017;356:93-103. https://doi.org/10.1016/j.heares.2017.10. 005.

4. Talbot KN, Hartley DE. Combined electro-acoustic stimulation: a beneficial union? Clin Otolaryngol. 2008;33(6):536-45 Epub 2009/01/08.
5. Helbig S, Rajan GP, Stover T, Lockley M, Kuthubutheen J, Green KM. Hearing preservation after cochlear reimplantation. Otol Neurotol. 2013;34(1):61-5 Epub 2012/12/04.

6. Jurawitz MC, Buchner A, Harpel T, Schussler M, Majdani O, Lesinski-Schiedat $A$, et al. Hearing preservation outcomes with different cochlear implant electrodes: nucleus(R) hybrid-L24 and nucleus freedom Cl422. Audiol Neurootol. 2014;19(5):293-309 Epub 2014/10/04.

7. Lenarz T, James C, Cuda D, Fitzgerald O'Connor A, Frachet B, Frijns JH, et al. European multi-centre study of the nucleus hybrid L24 cochlear implant. Int J Audiol. 2013;52(12):838-48 Epub 2013/09/03.

8. Suhling MC, Majdani O, Salcher R, Leifholz M, Buchner A, Lesinski-Schiedat $A$, et al. The impact of electrode array length on hearing preservation in cochlear implantation. Otol Neurotol. 2016;37(8):1006-15 Epub 2016/06/17.

9. Eshraghi AA, Gupta C, Van De Water TR, Bohorquez JE, Garnham C, Bas E, et al. Molecular mechanisms involved in cochlear implantation trauma and the protection of hearing and auditory sensory cells by inhibition of c-JunN-terminal kinase signaling. Laryngoscope. 2013;123(Suppl 1):S1-14 Epub 2013/02/06

10. Bas E, Gupta C, Van De Water TR. A novel organ of corti explant model for the study of cochlear implantation trauma. Anat Rec (Hoboken). 2012; 295(11):1944-56 Epub 2012/10/10.

11. Bas E, Dinh CT, Garnham C, Polak M, Van de Water TR. Conservation of hearing and protection of hair cells in cochlear implant patients' with residual hearing. Anat Rec (Hoboken). 2012;295(11):1909-27 Epub 2012/10/ 10.

12. Souza MEDCA, Costa KVTD, Vitorino PA, Bueno NB, Menezes PL. Effect of antioxidant supplementation on the auditory threshold in sensorineural hearing loss: a meta-analysis. Braz J Otorhinolaryngol. 2018;84(3):368-80. https://doi.org/10.1016/j.bjorl.2017.07.011.

13. Le Prell CG, Hughes LF, Miller JM. Free radical scavengers vitamins $A$, $C$, and E plus magnesium reduce noise trauma. Free Radic Biol Med. 2007;42(9): 1454-63.

14. Le Prell CG, Ojano-Dirain C, Rudnick EW, Nelson MA, DeRemer SJ, Prieskorn DM, et al. Assessment of nutrient supplement to reduce gentamicininduced ototoxicity. J Assoc Res Otolaryngol. 2014;15(3):375-93 Epub 2014/ 03/05.

15. Alvarado JC, Fuentes-Santamaria V, Melgar-Rojas P, Valero ML, Gabaldon-UII MC, Miller JM, et al. Synergistic effects of free radical scavengers and cochlear vasodilators: a new otoprotective strategy for age-related hearing loss. Front Aging Neurosci. 2015;7:86.

16. Le Prell CG, Gagnon PM, Bennett DC, Ohlemiller KK. Nutrient-enhanced diet reduces noise-induced damage to the inner ear and hearing loss. Transl Res. 2011;158(1):38-53.

17. Eastwood H, Pinder D, James D, Chang A, Galloway S, Richardson R, et al. Permanent and transient effects of locally delivered $n$-acetyl cysteine in a guinea pig model of cochlear implantation. Hear Res. 2010;259(1-2):24-30 Epub 2009/09/08.

18. NICE. Cochlear implants for children and adults with severe to profound deafness: technology appraisal guidance - TA1066. National Institute for Health and Clinical Excellence. 2009. [September 2019]; Available from: http://www.nice.org.uk/guidance/ta166.

19. Scheper V, Leifholz M, von der Leyen H, Keller M, Denkena U, Koch A, et al. ACEMg-mediated hearing preservation in cochlear implant patients receiving different electrode lengths (PROHEARING): study protocol for a randomized controlled trial. Trials. 2016;17:394 Epub 2016/08/10.

20. Lenarz T, Stover T, Buechner A, Lesinski-Schiedat A, Patrick J, Pesch J. Hearing conservation surgery using the hybrid-L electrode. Results from the first clinical trial at the Medical University of Hannover. Audiol Neurootol. 2009;14(Suppl 1):22-31 Epub 2009/04/29.

21. Cornelison TL, Clayton JA. Considering sex as a biological variable in biomedical research. Gender Genome. 2017;1 (2):89-93.

22. Wanna GB, O'Connell BP, Francis DO, Gifford RH, Hunter JB, Holder JT, et al. Predictive factors for short- and long-term hearing preservation in cochlear implantation with conventional-length electrodes. Laryngoscope. 2018; 128(2):482-9.

23. Zanetti D, Nassif N, Redaelli de Zinis LO. Factors affecting residual hearing preservation in cochlear implantation. Acta Otorhinolaryngol Ital. 2015;35(6): 433-41.

24. Santa Maria PL, Domville-Lewis C, Sucher CM, Chester-Browne R, Atlas MD. Hearing preservation surgery for cochlear implantation--hearing and quality of life after 2 years. Otol Neurotol. 2013;34(3):526-31. 
25. Gstoettner WK, Helbig S, Maier N, Kiefer J, Radeloff A, Adunka OF. Ipsilateral electric acoustic stimulation of the auditory system: results of long-term hearing preservation. Audiol Neurootol. 2006;11(Suppl 1):49-56 Epub 2006/ $10 / 26$.

26. Yao WN, Turner CW, Gantz BJ. Stability of low-frequency residual hearing in patients who are candidates for combined acoustic plus electric hearing. J Speech Lang Hear Res. 2006;49(5):1085-90.

27. Belenguer-Varea A, Tarazona-Santabalbina FJ, Avellana-Zaragoza JA, Ingles M, Mas-Bargues C, Martinez-Reig M. Oxidative stress and exceptional human longevity: systematic review. Free Radic Biol Med. 2019; Epub 2019/09/25.

\section{Publisher's Note}

Springer Nature remains neutral with regard to jurisdictional claims in published maps and institutional affiliations.

Ready to submit your research? Choose BMC and benefit from:

- fast, convenient online submission

- thorough peer review by experienced researchers in your field

- rapid publication on acceptance

- support for research data, including large and complex data types

- gold Open Access which fosters wider collaboration and increased citations

- maximum visibility for your research: over $100 \mathrm{M}$ website views per year

At $\mathrm{BMC}$, research is always in progress.

Learn more biomedcentral.com/submissions 